\title{
Safety and effectiveness of daily teriparatide in a prospective observational study in patients with osteoporosis at high risk of fracture in Japan: final report
}

\author{
This article was published in the following Dove Press journal: \\ Clinical Interventions in Aging \\ 6 July 2016 \\ Number of times this article has been viewed
}

\author{
Atsushi Nishikawa' \\ Takehiro Ishida ${ }^{2}$ \\ Masanori Taketsuna ${ }^{3}$ \\ Fumito Yoshiki ${ }^{4}$ \\ Hiroyuki Enomoto 4 \\ 'Global Patient Safety Japan, \\ Quality and Patient Safety, ${ }^{2}$ Clinical \\ Development Operations and \\ Innovations, Medicine Development \\ Unit Japan, ${ }^{3}$ Asia Pacific Statistical \\ Sciences, Medicine Development Unit \\ Japan, ${ }^{4}$ Medical Sciences, Medicines \\ Development Unit Japan, Eli Lilly Japan \\ K.K., Kobe, Japan
}

\begin{abstract}
This postmarketing surveillance study assessed the safety and effectiveness of teriparatide in patients with osteoporosis at high risk of fracture in Japan. The patients received teriparatide $20 \mu \mathrm{g}$ daily by subcutaneous injection, for a maximum of 24 months. Safety and effectiveness analyses were based on data from 1,847 patients who were predominantly female (92.6\%) with a mean age of 75.4 years. A total of 157 adverse drug reactions (ADRs) were reported in 140 (7.58\%) patients; the most common ADRs were hyperuricemia, nausea, and dizziness. Only six $(0.32 \%)$ patients reported serious ADRs, the most common being nausea (two patients; $0.1 \%$ ). Persistence with teriparatide treatment was $60.8 \%$ and $39.1 \%$ at 18 and 24 months, respectively. There were significant increases in biomarkers for bone formation (procollagen type I N-terminal propeptide and bone-specific alkaline phosphatase) and bone resorption (collagen type I crosslinked $\mathrm{C}$ telopeptide and tartrate-resistant acid phosphatase $5 \mathrm{~b}$ ) throughout the study. These were accompanied by significant increases in bone mineral density and low incidences of new vertebral and nonvertebral fractures. Patient-reported measurements for health-related quality of life revealed significant improvements from baseline in back pain and overall health-related quality of life (Short Form- $8^{\mathrm{TM}}$ health survey). The results of this 24-month postmarketing surveillance study imply that teriparatide has a favorable safety profile and is effective in the treatment of patients with osteoporosis at high risk of fracture in Japan. Teriparatide may also be a useful treatment for osteoporosis in other societies with aging populations.
\end{abstract}

Keywords: parathyroid hormone, bone biomarkers, bone mineral density, quality of life, safety

\section{Introduction}

Osteoporosis is a serious public health concern worldwide because of the morbidity and mortality associated with fragility fracture, ${ }^{1,2}$ which is expected to affect a large proportion of people $(40 \%-50 \%$ of women and $13 \%-22 \%$ of men) over the age of 50 years. ${ }^{3}$ In 2010 , osteoporosis was estimated to affect 27.6 million people in Europe. ${ }^{4}$ In Japan, the prevalence of osteoporosis is reported to be between $3.4 \%$ and $26.5 \%$ in people aged 40 years and over, and in 2009, osteoporosis was estimated to affect between 6.4 and 12.8 million people. ${ }^{5,6}$ In $2010,23 \%$ of the Japanese population were aged 65 years and over, and this is projected to increase to $32 \%$ in 2030 and to $40 \%$ by $2050 .^{7}$ The prevalence of osteoporosis in Japan is expected to increase due to the rapidly increasing proportion of elderly people.

Teriparatide (Forteo ${ }^{\circledR}$ [Eli Lilly and Company, Indianapolis, IN, USA]; recombinant 1-34 N-terminal sequence of human parathyroid hormone) is the first anabolic agent
Correspondence: Atsushi Nishikawa Global Patient Safety Japan, Quality and Patient Safety, Eli Lilly Japan K.K., 7-I-5 Isogamidori, Chuo-Ku, Kobe,

65I-0086, Japan

Tel +8I 782429917

$\mathrm{Fax}+81782429299$

Email nishikawa_atsushi@lilly.com 
approved for the treatment of patients with osteoporosis ${ }^{8}$ and has been reported to reduce the risk of fracture by increasing bone formation. ${ }^{9}$ The safety and efficacy of teriparatide has been assessed in randomized controlled trials (RCTs) and in observational studies conducted primarily in Caucasian populations. These studies have shown that teriparatide is well tolerated, reduces the risk of vertebral and nonvertebral fractures, and increases bone mineral density (BMD) and levels of bone turnover biomarkers in osteoporotic patients. ${ }^{10-12}$ In addition, the large-scale European Forsteo Observational Study (EFOS) demonstrated that teriparatide was effective in reducing fracture incidence in women with osteoporosis in real-world clinical practice. ${ }^{13,14}$

In Japan, teriparatide was approved in 2010 specifically for patients with osteoporosis at high risk of fracture, such as those with previous fracture, low BMD, or a family history of hip fracture. Double-blind RCTs have confirmed that osteoporotic patients in Japan had a similar response (increase in lumbar spine BMD) to teriparatide as Caucasian patients (10.4\% and $8.3 \%$ increase, respectively). ${ }^{12,15,16}$ A single bridging study and analysis of previous trial data demonstrated that body-weight-adjusted pharmacokinetics, changes in BMD, and safety profiles of teriparatide were similar in Japanese and non-Asian patients. ${ }^{16}$ However, no large, observational, postmarketing surveillance (PMS) studies have been conducted to confirm the effectiveness and safety of teriparatide in Japanese patients with osteoporosis. Such PMS studies are important because of differences in the way osteoporotic patients in Japan are treated and how they access services via the universal health insurance system. In addition, PMS studies assess the use of teriparatide in real-world conditions, including in patients with complex medical histories who may not be eligible for enrollment in RCTs. PMS of teriparatide is mandated by the Japan Ministry of Health, Labour and Welfare and will provide clinically relevant information on the use of teriparatide in a real-world clinical setting in Japan. The objectives of this PMS study were to assess the safety and effectiveness of teriparatide. The interim results of the study were reported previously, mainly focusing on patient characteristics and some interim outcomes; ${ }^{17}$ and we now report the final and definite results from the full study cohort of 1,847 patients over 24 months.

\section{Material and methods \\ Study design}

This was a prospective, multicenter, PMS study, with a 24-month observation period. A total of 1,882 patients were registered at 238 sites across Japan. The PMS study was conducted between October 29, 2010, and February 28, 2014, using a central registration method. The study was mandated by the Japan Ministry of Health, Labour and Welfare and was conducted in accordance with Good Post-marketing Study Practice of the Pharmaceutical Affairs Law of Japan. For this type of study, formal consent and institutional ethics approval were not required by our institutional IRB.

\section{Study population}

Participants were patients with osteoporosis who were at high risk of fracture, such as those with low BMD, previous fracture, older age, and/or family history of femoral neck fracture, in accordance with the prescribing information. ${ }^{8}$ Osteoporosis was diagnosed using diagnostic criteria for primary osteoporosis (year 2000 revision; Osteoporosis Diagnostic Criteria Review Committee of the Japanese Society for Bone and Mineral Research). ${ }^{18}$ Patients were excluded from the study if they had been previously prescribed teriparatide or if they had any contraindications to teriparatide treatment. ${ }^{8}$ The safety and effectiveness population, persistence population, and fracture incidence population included patients who had received at least one dose of teriparatide.

\section{Treatment protocol}

Patients were prescribed teriparatide $20 \mu \mathrm{g}$ daily $\left(\right.$ Forteo $^{\circledR}$ ), subcutaneously, for a maximum of 24 months. Clinical evaluations were performed at baseline and at 1, 3, 6, 12, 18 , and 24 months from the start of treatment. Patients who discontinued treatment before 24 months had a final clinical evaluation at the time of discontinuation (last visit).

\section{Safety profile}

Safety was assessed by reporting of adverse drug reactions (ADRs), defined as adverse events for which causal relation to teriparatide could not be excluded. All ADRs were reported by treating physicians. Recorded ADRs were classified using preferred terms and system organ classes according to the Medical Dictionary for Regulatory Activities (MedDRA; version 17.0, MedDRA MSSO, McLean, VA, USA).

\section{Persistence}

Persistence was calculated as the time from the start of treatment to the end of the 24-month study period or discontinuation and was based on physicians' reports. Discontinuation was defined as stopping teriparatide for at least 14 days or discontinued use of teriparatide.

\section{Effectiveness}

Effectiveness of teriparatide treatment was assessed by changes in biomarkers of bone turnover, BMD, fracture 
incidence, Visual Analog Scale (VAS) score for back pain, and Short Form-8 (SF-8 ${ }^{\mathrm{TM}}$ ) health survey score for healthrelated quality of life (HRQoL). Biomarkers of bone turnover were measured at baseline, 1, 3, and 6 months, and at treatment discontinuation. Biomarkers tested were as follows: serum procollagen type I N-terminal propeptide (PINP); serum bone-specific alkaline phosphatase (bone ALP); serum collagen type I cross-linked C telopeptide (CTX-I); and serum tartrate-resistant acid phosphatase 5b (TRACP5b). Each study site measured BMD according to their preferred method, at baseline and at 6-month intervals, at lumbar vertebrae 2-4 (L2-L4), femoral neck, and total hip. The number of new clinical fractures was counted at 6-monthly intervals. Incident vertebral and nonvertebral clinical fractures were defined as new fragility fractures that were reported at any postbaseline visit and were subsequently confirmed by radiographs at study sites. In accordance with the teriparatide Fracture Prevention Trial, ${ }^{12}$ nonvertebral fractures were defined as low energy fractures, except for pathological fractures. Patients rated the severity of their back pain at baseline, at 3, 12, 18, and 24 months and at treatment discontinuation, using the VAS score for pain, where a score of 0 indicates no back pain, and a score of 100 indicates worst possible back pain. Patients rated their HRQoL at the same observation time points as back pain, using the SF-8 health survey. ${ }^{19}$

\section{Statistical analysis}

Frequency and incidence were calculated for binary variables. Mean and standard deviation (SD) were calculated for continuous variables, except for percent changes from baseline in biomarkers of bone turnover (where first [Q1], second [median], and third [Q3] quartiles were also calculated) and BMD (where mean and 95\% confidence interval [CI] were calculated). Percent changes from baseline in biomarkers of bone turnover and BMD were assessed using the paired $t$-test; a $P$-value $<0.05$ was considered statistically significant. Persistence with teriparatide treatment and cumulative fracture rate were assessed using the Kaplan-Meier method. All statistical analyses were conducted using SAS Version 9.2 (SAS Institute, Cary, NC, USA).

\section{Results}

\section{Baseline characteristics and patient disposition}

A total of 1,882 patients were enrolled in the study between October 29, 2010, and February 29, 2012, and 1,847 were included in the safety and effectiveness analyses. Patients were excluded because their case report forms were not collectible $(n=22)$, there was a violation of their registration $(\mathrm{n}=10)$, or they did not attend a visit after the first prescription $(n=3)$. Most (92.6\%) included patients were female, with a mean (SD) age of 75.4 (9.1) years, and most (90.8\%) female patients were postmenopausal (Table 1). Approximately twothirds $(63.9 \%)$ of patients reported a history of fracture, and more than half $(52.1 \%)$ of patients experienced one or more vertebral fractures before registering in the study (Table 1).

Table I Baseline demographics, disease characteristics, comorbidities, and previous or concomitant osteoporosis treatments of patients with osteoporosis at high risk of fracture in Japan

\begin{tabular}{|c|c|}
\hline Characteristics & Patients $(\mathrm{N}=I, 847)$ \\
\hline \multicolumn{2}{|l|}{ Age } \\
\hline Mean (SD), years & $75.4(9.1)$ \\
\hline Median (min, max), years & $76(31,101)$ \\
\hline 30 to $<50$ years, $n(\%)$ & $19(1.0)$ \\
\hline 50 to $<65$ years, $\mathrm{n}(\%)$ & $210(11.4)$ \\
\hline 65 to $<75$ years, $n(\%)$ & $531(28.7)$ \\
\hline 75 to $<85$ years, $\mathrm{n}(\%)$ & $839(45.4)$ \\
\hline$\geq 85$ years, $\mathrm{n}(\%)$ & $248(13.4)$ \\
\hline Height, mean (SD), cm & | $48.0(7.8)$ \\
\hline Weight, mean (SD), kg & $47.3(8.9)$ \\
\hline Females, n (\%) & $1,711(92.6)$ \\
\hline \multicolumn{2}{|l|}{ Menopause status, n (\%) } \\
\hline Premenopause & $9(0.5)$ \\
\hline Postmenopause & $\mathrm{I}, 554(90.8)$ \\
\hline Unknown & $148(8.6)$ \\
\hline Age at menopause, mean (SD), years ${ }^{a}$ & $48.9(4.8)$ \\
\hline Current smoker, n (\%) & $35(1.9)$ \\
\hline Alcohol consumption (>3 U/d), n (\%) & $29(1.6)$ \\
\hline History of fracture, $n(\%)^{b}$ & $\mathrm{I}, 180(63.9)$ \\
\hline Past surgery for fracture, $\mathrm{n}(\%)$ & $243(13.2)$ \\
\hline $\begin{array}{l}\text { History of proximal femoral fracture in patients' } \\
\text { parents, } n(\%)^{b}\end{array}$ & $76(4.1)$ \\
\hline Vertebral fractures, n (\%) & $962(52.1)$ \\
\hline I & $370(20.0)$ \\
\hline 2 & $230(12.5)$ \\
\hline$\geq 3$ & $362(19.6)$ \\
\hline Nonvertebral fractures, n (\%) & $238(12.9)$ \\
\hline I & $191(10.3)$ \\
\hline 2 & $36(1.9)$ \\
\hline$\geq 3$ & II (0.6) \\
\hline \multicolumn{2}{|l|}{ BMD, \% of YAM, n (\%) } \\
\hline$\geq 80 \%$ & $133(7.2)$ \\
\hline 70 to $<80 \%$ & I $50(8.1)$ \\
\hline$<70 \%$ & $670(36.3)$ \\
\hline \multicolumn{2}{|l|}{ CKD ${ }^{b}$ stage, $n(\%)$} \\
\hline I & $194(10.5)$ \\
\hline 2 & $710(38.4)$ \\
\hline 3 & $350(18.9)$ \\
\hline 4 & $30(1.6)$ \\
\hline 5 & $3(0.2)$ \\
\hline
\end{tabular}

Notes: anformation on age at menopause was available for 598/I,554 postmenopausal female patients. 'Information was not available on history of fracture for 224 patients; history of femoral fracture in patients' parents for 872 patients; BMD for 894 patients; CKD for 560 patients; current smoking status for 410 patients; and alcohol consumption for 442 patients from the I,847 patient cohort.

Abbreviations: BMD, bone mineral density; CKD, chronic kidney disease; min, minimum; max, maximum; n, number; SD, standard deviation; YAM, young adult mean. 


\section{Comorbidities and previous/concomitant treatment for osteoporosis}

Nearly half (48.3\%) of patients included in the study reported one or more comorbidity (Table S1). The most frequently reported comorbidities were renal impairment $(21.9 \%)$, hypertension (16.6\%), hepatic impairment (11.7\%), and rheumatoid arthritis (8.2\%; Table S1). Secondary osteoporosis and glucocorticoid-induced osteoporosis were each reported in $8.0 \%$ of patients (Table S1). Approximately one-third (32.4\%) of patients had a past medical history (Table S1). Of the 1,847 patients in the study, more than half $(59.6 \%)$ had previously received treatment for osteoporosis; the remaining patients $(40.4 \%)$ were treatment naive. The most common treatments were alendronate $(26.7 \%)$, alfacalcidol $(16.8 \%)$, risedronate $(14.4 \%)$, and raloxifene $(10.3 \%)$ (Table S1). A total of $406(22.0 \%)$ patients were receiving concomitant treatment for osteoporosis during the study (Table S1).

\section{Safety}

A total of 157 ADRs were reported in 140 (7.58\%) patients (Table 2). The most commonly reported ADRs in patients

Table 2 Adverse drug reactions in patients with osteoporosis at high risk of fracture following treatment with teriparatide in Japan

\begin{tabular}{|c|c|}
\hline Adverse drug reaction & $\begin{array}{l}N=I, 847 \\
n(\%)\end{array}$ \\
\hline Patients experiencing adverse drug reactions & $140(7.58)$ \\
\hline Total adverse drug reactions reported & 157 \\
\hline \multicolumn{2}{|l|}{ Most commonly reported reactions ${ }^{\mathrm{a}}$} \\
\hline Hyperuricemia & $19(1.03)$ \\
\hline Nausea & $16(0.87)$ \\
\hline Dizziness & $10(0.54)$ \\
\hline Headache & $9(0.49)$ \\
\hline Increased blood alkaline phosphate & $7(0.38)$ \\
\hline Palpitations & $6(0.32)$ \\
\hline Hypercalcemia & $5(0.27)$ \\
\hline Rash & $5(0.27)$ \\
\hline Decreased appetite & $4(0.22)$ \\
\hline Renal impairment ${ }^{\mathrm{b}}$ & $4(0.22)$ \\
\hline Abdominal discomfort & $3(0.16)$ \\
\hline Eczema & $3(0.16)$ \\
\hline Pruritus & $3(0.16)$ \\
\hline Urticaria & $3(0.16)$ \\
\hline Feeling abnormal ${ }^{\mathrm{b}}$ & $3(0.16)$ \\
\hline Patients experiencing serious adverse drug reactions & $6(0.32)$ \\
\hline Total serious adverse drug reactions reported & 7 \\
\hline Nausea & $2(0.1 I)$ \\
\hline Constipation & I $(0.05)$ \\
\hline Hypercalcemia & I $(0.05)$ \\
\hline Toxic skin eruption & $\mathrm{I}(0.05)$ \\
\hline Calculus bladder & I $(0.05)$ \\
\hline Renal impairment & I (0.05) \\
\hline
\end{tabular}

Notes: ${ }^{a}$ Adverse drug reactions reported in $>2(0.1 \%)$ patients in the safety analysis. ${ }^{b}$ Adverse drug reactions that are not described in the usage notes for the study drug.

Abbreviation: $\mathrm{n}$, number. were hyperuricemia $(1.03 \%)$, nausea $(0.87 \%)$, dizziness $(0.54 \%)$, headache $(0.49 \%)$, and increased blood ALP $(0.38 \%)$ (Table 2$)$. Only seven serious ADRs were reported in six patients; these were nausea, reported in two $(0.11 \%)$ patients, and constipation, hypercalcemia, toxic skin eruption, calculus bladder, and renal impairment, each reported in one $(0.05 \%)$ patient (Table 2$)$.

\section{Persistence with teriparatide treatment}

Overall, $60.8 \%$ of patients persisted with teriparatide treatment for the first 18 months and $39.1 \%$ persisted for the first 24 months (Figure 1). A total of 977 (52.9\%) patients discontinued treatment, most commonly because of patient decision (341 patients; $18.5 \%$ ), doctor decision (233 patients; $12.6 \%$ ), adverse events (131 patients; $7.1 \%$ ), end of treatment (patients considered by the investigator to no longer require treatment with teriparatide; 133 patients; $7.2 \%$ ), lost to follow-up (120 patients; $6.5 \%$ ), or insufficient effect (four patients; $0.2 \%)$. A total of 15 patients $(0.8 \%)$ discontinued because of death; these deaths were considered (by the investigators) to be unrelated to teriparatide treatment.

\section{Biomarkers for bone turnover}

The levels of biomarkers for bone formation (PINP and bone ALP) were significantly increased from baseline at all time points during the study (Figure 2A and B). The median (Q1, Q3) percent change from baseline at the last observation carried forward (LOCF) was 208.9\% $(91.0,454.7 ; \mathrm{n}=449)$ for PINP and $51.35 \%(14.1,99.3 ; \mathrm{n}=145)$ for bone ALP. The levels of biomarkers for bone resorption (CTX-I and TRACP5b)

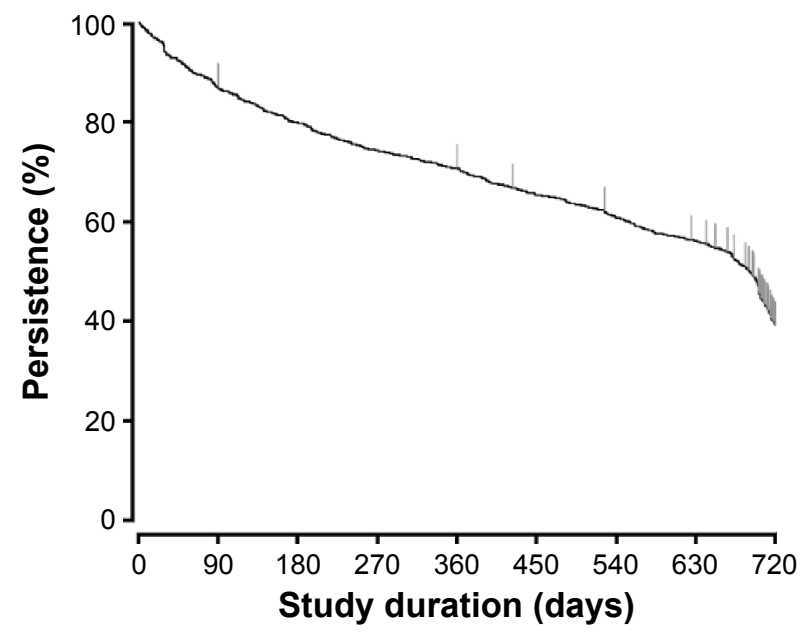

Persistence

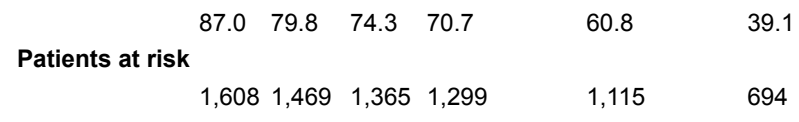

Figure I Kaplan-Meier curve of persistence with teriparatide treatment in patients with osteoporosis at high risk of fracture in Japan. 

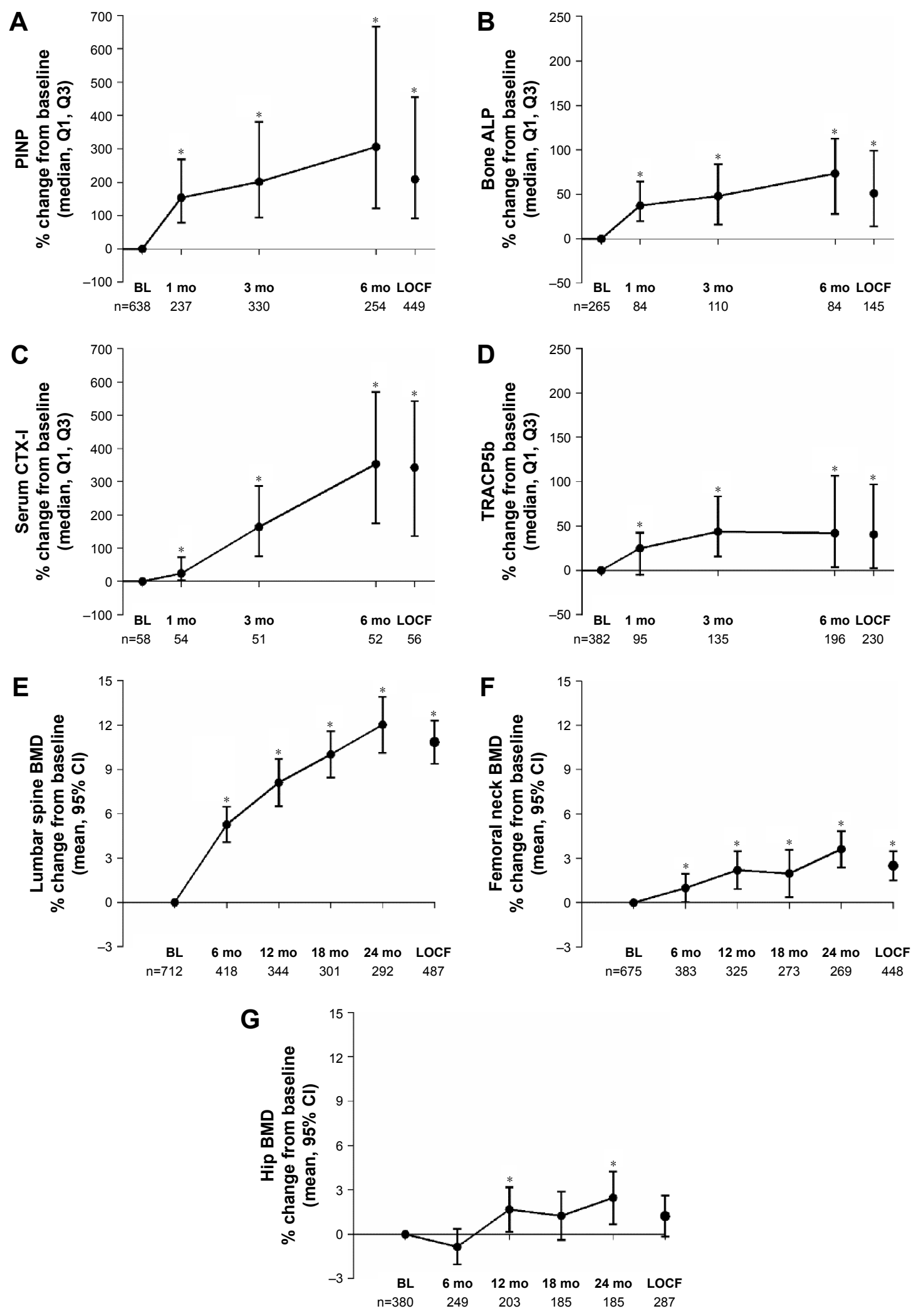

Figure 2 Percent change from baseline in biomarkers of bone turnover (median, QI, Q3) and BMD (mean, $95 \% \mathrm{Cl}$ ) after up to 24 months of teriparatide treatment and at LOCF in patients with osteoporosis at high risk of fracture in Japan.

Notes: Biomarkers for bone formation were PINP $(\mathbf{A})$ and bone ALP $(\mathbf{B})$ and for bone resorption were CTX-I (C) and TRACP5b (D). The mean (SD) baseline values were: PINP 5 I.49 (59.49) ng/mL; bone ALP 19.37 (17.45) IU/L; CTX-I $0.22(0.16) \mathrm{ng} / \mathrm{mL}$; and TRACP5b $45 \mathrm{I} .50$ (245.29) $\mathrm{mU} / \mathrm{dL}$. BMD was measured at the lumbar spine (E), femoral neck (F), and total hip $(\mathbf{G}) . * P<0.05$ for the increase from baseline at each time point.

Abbreviations: bone ALP, bone-specific alkaline phosphatase; BL, baseline; BMD, bone mineral density; Cl, confidence interval; CTX-I, collagen type I cross-linked C telopeptide; LOCF, last observation carried forward; PINP, procollagen type I N-terminal propeptide; QI, first quartile; Q3, third quartile; SD, standard deviation; TRACP5b, tartrate-resistant acid phosphatase $5 b$. 
A

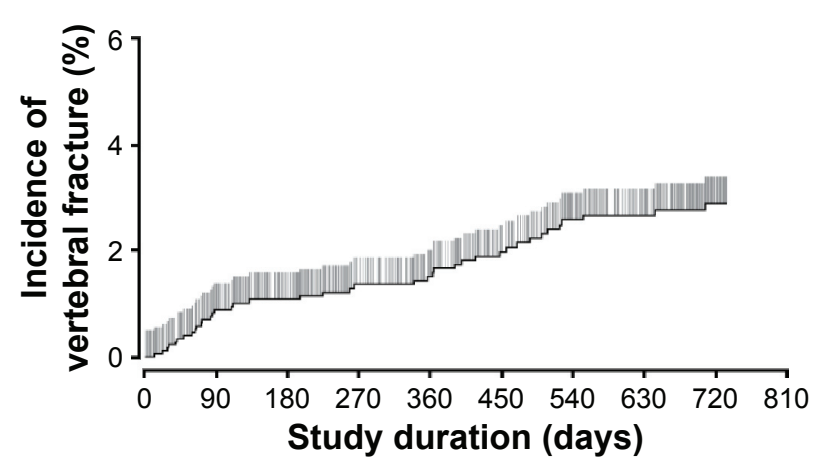

Incidence (\%)

Patients at risk (n)

$\begin{array}{lllll}0.88 & 1.08 & 1.36 & 1.51 & 2.58\end{array}$

$\begin{array}{lllll}1,600 & 1,465 & 1,357 & 1,292 & 1,102\end{array}$
B

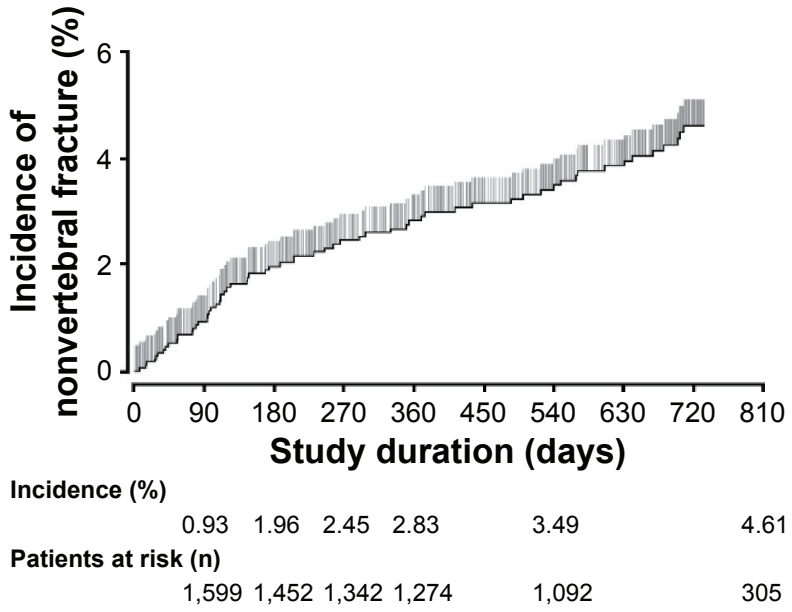

Figure 3 Kaplan-Meier estimate of vertebral $(\mathbf{A})$ and nonvertebral $(\mathbf{B})$ fractures in patients with osteoporosis at high risk of fracture during treatment with teriparatide in Japan.

were also significantly increased from baseline at all time points during the study (Figure $2 \mathrm{C}$ and $\mathrm{D}$ ). The median (Q1, Q3) percent changes at the LOCF was 342.5\% (135.3, $541.9 ; \mathrm{n}=56)$ for CTX-I and $40.4 \%(2.5,96.9 ; \mathrm{n}=230)$ for TRACP5b.

\section{BMD}

At baseline, the mean (95\% CI) BMD was $0.731(0.711-$ $0.751) \mathrm{g} / \mathrm{cm}^{2}$ at the lumbar spine, $0.541(0.533-0.549) \mathrm{g} / \mathrm{cm}^{2}$ at the femoral neck, and $0.563(0.551-0.575) \mathrm{g} / \mathrm{cm}^{2}$ at the total hip. There were significant increases from baseline at all time points at the lumbar spine (L2-L4) and femoral neck and at 12 and 24 months at the total hip (Figure 2E-G). The greatest increases were observed at the lumbar spine, where the mean $(95 \% \mathrm{CI})$ percent change from baseline was 5.3\% (4.1\%-6.5\%, $\mathrm{n}=418), 8.1 \%(6.5 \%-9.7 \%, \mathrm{n}=344), 10.0 \%$ $(8.5 \%-11.6 \%, \mathrm{n}=301), 12.0 \%(10.1 \%-13.9 \%, \mathrm{n}=292)$, and $10.9 \%(9.4 \%-12.3 \%, \mathrm{n}=487)$ at $6,12,18$, and 24 months, and at the LOCF, respectively.

\section{Fracture incidence}

The incidence of new fractures with increasing treatment duration is shown in Figure 3. Overall, the cumulative incidence of new vertebral or nonvertebral fracture during the 24 -month study was $2.9 \%$ and $4.6 \%$, respectively.

\section{Back pain}

Back pain significantly $(P<0.001)$ improved from baseline at all time points during the study (Figure S1). The mean (SD) back pain VAS score at baseline was 41.8 (28.4). The mean ( $95 \% \mathrm{CI})$ change from baseline in back pain VAS scores at
$3,12,18$, and 24 months and at the LOCF were $-10.0(-12.0$ to -8.0$),-12.5$ ( -14.7 to -10.2$),-12.9(-15.5$ to -10.4$),-13.8$ (-16.6 to -11.0$)$, and $-13.1(-15.1$ to -11.1$)$, respectively.

\section{HRQoL}

Overall, SF-8 health survey scores significantly improved following treatment with teriparatide (Figure S2). The mean (SD) SF-8 scores at baseline ranged from 38.38 (9.32) for the physical component summary score to 46.22 (8.21) for the mental health domain. The mean $(95 \% \mathrm{CI})$ change from baseline to the LOCF in SF-8 scores ranged from 2.37 (1.72-3.01) for the mental component summary score to 4.50 (3.73-5.27) for the role physical domain.

\section{Discussion}

In this first large-scale PMS study in Japan, teriparatide was shown to have favorable safety and effectiveness profiles in osteoporotic patients at high risk of fracture. Importantly, teriparatide was well tolerated, with no new clinically significant safety concerns identified, and persistence with teriparatide treatment was similar to or higher than that reported in other studies. ${ }^{20-23}$ Early significant increases in bone formation biomarkers were followed by subsequent increases in bone resorption biomarkers. The cumulative incidence of new vertebral and nonvertebral fracture to 24 months was $2.9 \%$ and $4.6 \%$, respectively, and patient-reported back pain and overall HRQoL improved significantly. To alleviate the health-related burden of Japan's rapidly aging population, the Japanese Government established mandatory long-term public health insurance in 2000. ${ }^{24}$ Under this insurance, recipients can select the nursing care services required for 
the treatment of osteoporosis and its associated comorbidities, leading to possible serious socioeconomic concerns. Therefore, information gained from this PMS study will provide an essential reference for the use of teriparatide, with its favorable tolerability profile, to prevent subsequent fragility fracture in osteoporotic patients in Japan. The results from this PMS study will also be relevant to many other countries where teriparatide can be used for patients at high risk of fracture.

The safety profile for teriparatide in this PMS study is consistent with that of Japanese populations reported in previous RCTs ${ }^{15,25}$ and of Caucasian populations reported in previous RCTs and observational studies. ${ }^{12,21,26,27}$ Overall, teriparatide treatment was well tolerated, with $7.1 \%$ of patients discontinuing because of adverse events, consistent with the rates reported in other studies of teriparatide. ${ }^{12,15,20,25,26}$ In addition, $7.58 \%$ of patients experienced ADRs (most commonly hyperuricemia, nausea, and dizziness), and only $0.32 \%$ of patients experienced serious ADRs. There were no reports of osteosarcoma, in agreement with the 7-year findings from a 15-year surveillance study of teriparatide in the United States. ${ }^{28}$ A bridging study that analyzed previous trial data also reported no new safety concerns in Japanese women treated with teriparatide compared with Caucasian women. ${ }^{16}$ However, a greater proportion of the Japanese women in that study reported ADRs compared with this PMS study, possibly due to the more sensitive screening for ADRs in the bridging study analysis. ${ }^{15,16}$ Importantly, the favorable safety results of this PMS study were observed in a patient population in which almost $60 \%$ were aged $\geq 75$ years. Similarly, in EFOS, teriparatide was well tolerated, with no significant differences in the safety profiles of patients aged $<75$ years compared with those aged $\geq 75$ years. ${ }^{26}$

Poor adherence and persistence with osteoporosis treatments are major issues faced by physicians. ${ }^{29}$ Although administration of teriparatide requires daily self-injection, the persistence rates in this PMS study were $70.7 \%, 60.8 \%$, and $39.1 \%$ of patients at 12,18 , and 24 months, respectively. Persistence at 12 and 18 months was similar to that reported previously in Japan and Europe, ${ }^{20-22}$ whereas persistence at 24 months was greater than that reported in the United States. ${ }^{23}$ The persistence rates we observed may have resulted, in part, from the patient support and educational programs that were part of the treatment program. ${ }^{20,30}$ In addition, the comprehensive public health insurance system for the elderly population in Japan may provide the financial support needed to facilitate persistence with treatment. Persistence with teriparatide treatment has been demonstrated to be associated with lower risk of fracture. ${ }^{31}$ Although direct associations between treatment persistence and effectiveness were not investigated in this study, the high rate of persistence was likely to have contributed to the lower fracture risk and the improvement of BMD, back pain, and HRQoL compared with studies conducted in other countries.

The increases in BMD observed at the lumbar spine, femoral neck, and total hip in this PMS study were similar to those observed in an RCT conducted in Japanese patients ${ }^{15}$ and an observational study conducted in Europe. ${ }^{27}$ There was a large increase in lumbar spine BMD at 6 months, which then steadily increased over the next 18 months. In addition, the transient decrease in BMD at the total hip observed at 6 months in this PMS study is consistent with previous reports on patients who had been treated with antiresorptive therapy prior to teriparatide ${ }^{32,33}$ and is thought to occur because of the matrix remodeling of highly mineralized bone caused by antiresorptives. ${ }^{33}$ In contrast, no such decrease in BMD was observed in the pivotal fracture prevention trial conducted in treatment-naive patients. ${ }^{12}$ Given that approximately $40 \%$ of the patients in this PMS study were treatment naive and that $46 \%$ had received previous treatment with a bisphosphonate, the transient decrease in hip BMD is not unexpected.

Teriparatide stimulates bone turnover while maintaining a positive balance between bone formation and bone resorption, with associated increases in biomarkers for both processes. ${ }^{34}$ Bone biomarkers are clinically useful measurements in the earlier stages of treatment when BMD data are less informative. ${ }^{35}$ Previous studies have demonstrated a correlation between an early change in PINP level and a later increase in BMD during teriparatide treatment. ${ }^{36,37}$ In the current study, the early increase in the bone formation biomarkers PINP and bone ALP, and the later increase in bone resorption biomarkers, are consistent with previous reports of teriparatide in Japan ${ }^{15,25}$ and with those reported in largely Caucasian study populations..$^{38-40}$ One novel finding of this PMS study is the time course analysis of serum TRACP5b levels, which demonstrated an increase in response to teriparatide as early as 1 and 3 months. Whereas CTX-I reflects osteoclastic activity and has a prominent circadian rhythm, TRACP5b reflects the number of osteoclasts and is essentially independent of time. ${ }^{41}$ In our study, TRACP5b levels plateaued at approximately 50\% above baseline levels at 3 and 6 months, which together with the greater percentage change in PINP, indicates an overall positive balance of bone remodeling associated with teriparatide treatment.

The improvement in bone microarchitecture and bone strength with teriparatide is thought to translate to a 
reduced risk of fracture in postmenopausal women with osteoporosis. ${ }^{9,12}$ In both the pivotal fracture prevention trial and EFOS, teriparatide $(20 \mu \mathrm{g})$ treatment for up to 36 months significantly reduced the risk of new vertebral and nonvertebral fractures. ${ }^{12,13}$ In the current study, the incidences of new vertebral and nonvertebral fractures during the 24-month period were $2.9 \%$ and $4.6 \%$, respectively. Direct comparison of fracture incidence among different studies is problematic because of differences in study design and patient populations. However, the fracture incidences in our study were lower than those reported in previous studies, ${ }^{12,13}$ possibly because of the low number of patients with previous fracture history in this PMS study, despite their older age. ${ }^{21}$ In addition, our results are similar to those reported in an RCT of osteoporotic patients in Japan, in which $4.4 \%$ and $2.2 \%$ of patients experienced new vertebral and nonvertebral fractures, respectively, during the 12-month teriparatide treatment period, and no new fractures were observed during the 12 months following treatment. ${ }^{15}$

In addition to an increased risk of fracture, postmenopausal women with osteoporosis have an increased risk of associated complications such as chronic back pain that can lead to reduced HRQoL. ${ }^{42}$ This is the first longitudinal study (24 months treatment) in osteoporotic patients in Japan to show substantial improvement in HRQoL following teriparatide treatment. Patients reported a significant decrease in the severity of back pain from 3 months onwards, which was accompanied by significant improvement in overall SF-8 scores, with the greatest improvements in the role physical and bodily pain domains. Despite some differences in the patients' characteristics, our findings on back pain and HRQoL were consistent with those in EFOS, which further demonstrated that improvements in back pain persist for at least 18 months after teriparatide treatment is terminated. ${ }^{14}$

Interpretation of the findings from this study is limited by the biases that can be inherent in observational studies. The study involved a single cohort, patients were not randomized by age or treatment group, and there was no control or comparator group. The effectiveness of teriparatide was not compared between patients with or without previous treatment in this study. In addition, not all patients had information available on biomarkers of bone turnover, BMD, and HRQoL measurements at all of the study time points. Importantly, however, the strength of the study is that the population reflected real-world clinical practice for osteoporotic patients in Japan. The study included a large sample size with no age or sex restrictions, and a considerable proportion of patients had comorbidities and had either previously been treated for osteoporosis or were receiving concurrent osteoporosis treatment at the time of the study. Although the patients in this PMS study were older and had less severe baseline characteristics in terms of fracture history and risk, BMD, and back pain compared with previous studies in Caucasian populations, there were no new safety concerns and similar clinical benefits of teriparatide treatment were observed. ${ }^{14,31,43-45}$ This study highlights both the importance of PMS studies in real-world settings and the need for patient education on available treatments and their HRQoL benefits. The findings are clinically relevant because Japan is more affected by population aging than any other country. ${ }^{24}$ Finally, patients were administered teriparatide in accordance with its Japanese indication. Thus, our results illustrate the real-world experience in Japan where, owing to the highly developed public health insurance system, physicians prescribe teriparatide strictly according to the indication.

\section{Conclusion}

In conclusion, this PMS study demonstrated that teriparatide has favorable safety and effectiveness profiles including HRQoL in osteoporotic patients at high risk of fracture in Japan. Importantly, these results were obtained in a patient population that was older, had more comorbidities, less severe fracture history, and more treatment-naïve patients than populations used in previous RCTs.

\section{Acknowledgments}

The authors acknowledge the efforts of the investigators who participated in the study and thank the contributors to manuscript preparation, editing, and review (Hideaki Katagiri and Mika Tsujimoto from Eli Lilly Japan). This study was sponsored by Eli Lilly Japan, manufacturer/licensee of teriparatide $\left(\right.$ Forteo $^{\circledR}$ ). Medical writing assistance was provided by Serina Stretton, PhD, CMPP, and Rebecca Lew, PhD, CMPP of ProScribe - Envision Pharma Group, and was funded by Eli Lilly. ProScribe's services complied with international guidelines for Good Publication Practice (GPP3).

\section{Author contributions}

All authors were involved in the study design, data collection, data interpretation, and statistical analysis, and contributed to the drafting of the manuscript.

\section{Disclosure}

AN, MT, FY, and HE are employees of Eli Lilly Japan K.K. TI was an employee of Eli Lilly Japan K.K. during the study and the preparation of this manuscript and is currently an 
employee of Bristol-Myers Squibb K.K. Eli Lilly Japan was involved in the study design, data collection, data analysis, and preparation of the manuscript. The authors report no other conflicts of interest in this work.

\section{References}

1. Johnell O, Kanis JA. An estimate of the worldwide prevalence, mortality and disability associated with hip fracture. Osteoporos Int. 2004; 15(11):897-902.

2. Nazrun AS, Tzar MN, Mokhtar SA, Mohamed IN. A systematic review of the outcomes of osteoporotic fracture patients after hospital discharge: morbidity, subsequent fractures, and mortality. Ther Clin Risk Manag. 2014;10:937-948.

3. Johnell O, Kanis J. Epidemiology of osteoporotic fractures. Osteoporos Int. 2005;16(Suppl 2):S3-S7.

4. Hernlund E, Svedbom A, Ivergard M, et al. Osteoporosis in the European Union: medical management, epidemiology and economic burden. A report prepared in collaboration with the International Osteoporosis Foundation (IOF) and the European Federation of Pharmaceutical Industry Associations (EFPIA). Arch Osteoporos. 2013;8:136.

5. Orimo H, Nakamura T, Hosoi T, et al. Japanese 2011 guidelines for prevention and treatment of osteoporosis - executive summary. Arch Osteoporos. 2012;7:3-20.

6. Yoshimura N, Muraki S, Oka H, et al. Prevalence of knee osteoarthritis, lumbar spondylosis, and osteoporosis in Japanese men and women: the research on osteoarthritis/osteoporosis against disability study. J Bone Miner Metab. 2009;27(5):620-628.

7. Hagino H. Features of limb fractures: a review of epidemiology from a Japanese perspective. J Bone Miner Metab. 2007;25(5):261-265.

8. Forteo ${ }^{\circledR}$ (teriparatide [rDNA origin] injection) [prescribing information]. Kobe, Japan: Eli Lilly Japan K.K.; 2014.

9. Hodsman AB, Bauer DC, Dempster DW, et al. Parathyroid hormone and teriparatide for the treatment of osteoporosis: a review of the evidence and suggested guidelines for its use. Endocr Rev. 2005;26(5):688-703.

10. Krege JH, Wan X. Teriparatide and the risk of nonvertebral fractures in women with postmenopausal osteoporosis. Bone. 2012;50(1):161-164

11. Kurland ES, Cosman F, McMahon DJ, Rosen CJ, Lindsay R, Bilezikian JP. Parathyroid hormone as a therapy for idiopathic osteoporosis in men: effects on bone mineral density and bone markers. J Clin Endocrinol Metab. 2000;85(9):3069-3076.

12. Neer RM, Arnaud CD, Zanchetta JR, et al. Effect of parathyroid hormone (1-34) on fractures and bone mineral density in postmenopausal women with osteoporosis. N Engl J Med. 2001;344(19):1434-1441.

13. Fahrleitner-Pammer A, Langdahl BL, Marin F, et al. Fracture rate and back pain during and after discontinuation of teriparatide: 36-month data from the European Forsteo Observational Study (EFOS). Osteoporos Int. 2011;22(10):2709-2719.

14. Walsh JB, Lems WF, Karras D, et al. Effectiveness of teriparatide in women over 75 years of age with severe osteoporosis: 36-month results from the European Forsteo Observational Study (EFOS). Calcif Tissue Int. 2012;90(5):373-383.

15. Miyauchi A, Matsumoto T, Sugimoto T, Tsujimoto M, Warner MR, Nakamura T. Effects of teriparatide on bone mineral density and bone turnover markers in Japanese subjects with osteoporosis at high risk of fracture in a 24-month clinical study: 12-month, randomized, placebo-controlled, double-blind and 12-month open-label phases. Bone. 2010;47(3):493-502.

16. Tsujimoto M, Uenaka K, Iwata A, Higashiuchi Y, Sowa H. Effects of teriparatide in Japanese and non-Japanese populations: bridging findings on pharmacokinetics and efficacy. J Bone Miner Metab. 2012;30(3): 326-337.

17. Yamamoto T, Taketsuna M, Guo X, Sato M, Sowa H. The safety and effectiveness profile of daily teriparatide in a prospective observational study in Japanese patients with osteoporosis at high risk for fracture: interim report. J Bone Miner Metab. 2014;32(6):699-708.
18. Orimo H, Hayashi Y, Fukunaga M, et al; Osteoporosis Diagnostic Criteria Review Committee: Japanese Society for Bone and Mineral Research. Diagnostic criteria for primary osteoporosis: year 2000 revision. J Bone Miner Metab. 2001;19(6):331-337.

19. Ware JE, Kosinski M, Dewey JE, Gandek B. How to Score and Interpret Single-Item Health Status Measures: a Manual for Users of the SF-8 Health Survey. Lincoln, RI: Quality Metric Inc; 2001.

20. Arden NK, Earl S, Fisher DJ, Cooper C, Carruthers S, Goater M. Persistence with teriparatide in patients with osteoporosis: the UK experience. Osteoporos Int. 2006;17(11):1626-1629.

21. Langdahl BL, Rajzbaum G, Jakob F, et al. Reduction in fracture rate and back pain and increased quality of life in postmenopausal women treated with teriparatide: 18-month data from the European Forsteo Observational Study (EFOS). Calcif Tissue Int. 2009;85(6): 484-493.

22. Tanaka I, Sato M, Sugihara T, et al. Adherence and persistence with once-daily teriparatide in Japan: a retrospective, prescription database, cohort study. J Osteoporos. 2013;2013:654218.

23. Yu S, Burge RT, Foster SA, Gelwicks S, Meadows ES. The impact of teriparatide adherence and persistence on fracture outcomes. Osteoporos Int. 2012;23(3):1103-1113.

24. Tamiya N, Noguchi H, Nishi A, et al. Population ageing and wellbeing: lessons from Japan's long-term care insurance policy. Lancet. 2011; 378(9797):1183-1192.

25. Miyauchi A, Matsumoto T, Shigeta H, Tsujimoto M, Thiebaud D, Nakamura T. Effect of teriparatide on bone mineral density and biochemical markers in Japanese women with postmenopausal osteoporosis: a 6-month dose-response study. J Bone Miner Metab. 2008; 26(6):624-634.

26. Boonen S, Marin F, Mellstrom D, et al. Safety and efficacy of teriparatide in elderly women with established osteoporosis: bone anabolic therapy from a geriatric perspective. J Am Geriatr Soc. 2006;54(5):782-789.

27. Obermayer-Pietsch BM, Marin F, McCloskey EV, et al; EUROFORS Investigators. Effects of two years of daily teriparatide treatment on BMD in postmenopausal women with severe osteoporosis with and without prior antiresorptive treatment. J Bone Miner Res. 2008; 23(10):1591-1600.

28. Andrews EB, Gilsenan AW, Midkiff K, et al. The US postmarketing surveillance study of adult osteosarcoma and teriparatide: study design and findings from the first 7 years. J Bone Miner Res. 2012;27(12): 2429-2437.

29. Huybrechts KF, Ishak KJ, Caro JJ. Assessment of compliance with osteoporosis treatment and its consequences in a managed care population. Bone. 2006;38(6):922-928.

30. Briot K, Ravaud P, Dargent-Molina P, Zylberman M, Liu-Leage S, Roux C. Persistence with teriparatide in postmenopausal osteoporosis; impact of a patient education and follow-up program: the French experience. Osteoporos Int. 2009;20(4):625-630.

31. Rajzbaum G, Grados F, Evans D, Liu-Leage S, Petto H, AugendreFerrante B. Treatment persistence and changes in fracture risk, back pain, and quality of life amongst patients treated with teriparatide in routine clinical care in France: results from the European Forsteo Observational Study. Joint Bone Spine. 2014;81(1):69-75.

32. Boonen S, Marin F, Obermayer-Pietsch B, et al. Effects of previous antiresorptive therapy on the bone mineral density response to two years of teriparatide treatment in postmenopausal women with osteoporosis. J Clin Endocrinol Metab. 2008;93(3):852-860.

33. Ettinger B, San Martin J, Crans G, Pavo I. Differential effects of teriparatide on BMD after treatment with raloxifene or alendronate. J Bone Miner Res. 2004;19(5):745-751.

34. Compston JE. Skeletal actions of intermittent parathyroid hormone: effects on bone remodelling and structure. Bone. 2007;40(6):1447-1452.

35. Terreni A, Pezzati P. Biochemical markers in the follow-up of the osteoporotic patients. Clin Cases Miner Bone Metab. 2012;9(2):80-84.

36. Niimi R, Kono T, Nishihara A, et al. An algorithm using the early changes in PINP to predict the future BMD response for patients treated with daily teriparatide. Osteoporos Int. 2014;25(1):377-384. 
37. Tsujimoto M, Chen P, Miyauchi A, Sowa H, Krege JH. PINP as an aid for monitoring patients treated with teriparatide. Bone. 2011;48(4): 798-803.

38. Blumsohn A, Marin F, Nickelsen T, et al; EUROFORS Study Group. Early changes in biochemical markers of bone turnover and their relationship with bone mineral density changes after 24 months of treatment with teriparatide. Osteoporos Int. 2011;22(6):1935-1946.

39. Krege JH, Lane NE, Harris JM, Miller PD. PINP as a biological response marker during teriparatide treatment for osteoporosis. Osteoporos Int. 2014;25(9):2159-2171.

40. McClung MR, San Martin J, Miller PD, et al. Opposite bone remodeling effects of teriparatide and alendronate in increasing bone mass. Arch Intern Med. 2005;165(15):1762-1768.

41. Henriksen K, Tanko LB, Qvist P, Delmas PD, Christiansen C, Karsdal MA. Assessment of osteoclast number and function: application in the development of new and improved treatment modalities for bone diseases. Osteoporos Int. 2007;18(5):681-685.
42. Ljunggren O, Barrett A, Stoykov I, et al. Effective osteoporosis treatment with teriparatide is associated with enhanced quality of life in postmenopausal women with osteoporosis: the European Forsteo Observational Study. BMC Musculoskelet Disord. 2013;14:251.

43. Foster SA, Foley KA, Meadows ES, et al. Characteristics of patients initiating teriparatide for the treatment of osteoporosis. Osteoporos Int. 2008;19(3):373-377.

44. Ljunggren O, Benhamou CL, Dekker J, et al. Study description and baseline characteristics of the population enrolled in a multinational observational study of extended teriparatide use (ExFOS). Curr Med Res Opin. 2014;30(8):1607-1616.

45. Rajzbaum G, Jakob F, Karras D, et al. Characterization of patients in the European Forsteo Observational Study (EFOS): postmenopausal women entering teriparatide treatment in a community setting. Curr Med Res Opin. 2008;24(2):377-384. 


\section{Supplementary materials}

Table SI Baseline comorbidities and previous or concomitant osteoporosis treatments of patients with osteoporosis at high risk of fracture in Japan

\begin{tabular}{|c|c|}
\hline Characteristic & $\begin{array}{l}N=I, 847 \\
n(\%)\end{array}$ \\
\hline Comorbidities & $893(48.3)$ \\
\hline \multicolumn{2}{|l|}{ Most frequently occurring comorbidities } \\
\hline Hypertension & $306(16.6)$ \\
\hline Rheumatoid arthritis & $|5|(8.2)$ \\
\hline Hyperlipidemia & $140(7.6)$ \\
\hline Osteoarthritis & $105(5.7)$ \\
\hline Constipation & $97(5.3)$ \\
\hline Gastroesophageal reflux disease & $94(5.1)$ \\
\hline Insomnia & $86(4.7)$ \\
\hline Lumbar spinal canal stenosis & $83(4.5)$ \\
\hline Type 2 diabetes mellitus & $77(4.2)$ \\
\hline Gastritis & $66(3.6)$ \\
\hline Renal impairment ${ }^{\mathrm{a}}$ & $404(21.9)$ \\
\hline Hepatic impairment ${ }^{\mathrm{b}}$ & $217(11.7)$ \\
\hline Secondary osteoporosis ${ }^{c}$ & $148(8.0)$ \\
\hline Glucocorticoid-induced osteoporosis $^{c}$ & $148(8.0)$ \\
\hline Past medical history & $598(32.4)$ \\
\hline \multicolumn{2}{|l|}{ Most frequently reported medical history } \\
\hline Vertebral compression fracture & $58(3.1)$ \\
\hline Uterine leiomyoma & $5 I(2.8)$ \\
\hline Appendicitis & $41(2.2)$ \\
\hline Cerebral infarction & $35(1.9)$ \\
\hline Femoral neck fracture & $28(1.5)$ \\
\hline Gastric ulcer & $27(1.5)$ \\
\hline Hypertension & $25(1.4)$ \\
\hline Osteoarthritis & $22(1.2)$ \\
\hline Lumbar spinal canal stenosis & $21(1.1)$ \\
\hline Cataract & $21(1.1)$ \\
\hline History of drug therapy for osteoporosis & $\mathrm{I}, 10 \mathrm{I}(59.6)$ \\
\hline \multicolumn{2}{|c|}{ Most common previous drug therapy for osteoporosis (used by $\geq 5$ patients) } \\
\hline Alendronate (oral) & $493(26.7)$ \\
\hline Alfacalcidol (oral) & $311(16.8)$ \\
\hline Risedronate (oral) & $266(14.4)$ \\
\hline Raloxifene (oral) & $190(10.3)$ \\
\hline Elcatonin (intramuscular) & $113(6.1)$ \\
\hline Minodronic acid (oral) & $89(4.8)$ \\
\hline Menatetrenone (oral) & $58(3.1)$ \\
\hline Calcitriol (oral) & $52(2.8)$ \\
\hline L-Aspartic acid (oral) & $34(1.8)$ \\
\hline Eldecalcitol (oral) & $18(1.0)$ \\
\hline Bazedoxifene (oral) & $15(0.8)$ \\
\hline Concomitant drug therapy for osteoporosis & $406(22.0)$ \\
\hline \multicolumn{2}{|c|}{ Most common concomitant drug therapy for osteoporosis (used by $\geq 5$ patients) } \\
\hline L-aspartic acid (oral) & $176(9.5)$ \\
\hline Alfacalcidol (oral) & $145(7.9)$ \\
\hline Menatetrenone (oral) & $101(5.5)$ \\
\hline Eldecalcitol (oral) & $39(2.1)$ \\
\hline Elcatonin (intramuscular) & $34(1.8)$ \\
\hline Raloxifene (oral) & $31(1.7)$ \\
\hline Risedronate (oral) & $28(1.5)$ \\
\hline Alendronate (oral) & $13(0.7)$ \\
\hline Minodronic acid (oral) & $10(0.5)$ \\
\hline Bazedoxifene (oral) & $7(0.4)$ \\
\hline
\end{tabular}

Notes: a Patients with renal impairment or chronic kidney disease stages 3 to 5 are defined as having renal impairment. ${ }^{b}$ Patients with liver impairment or abnormal laboratory data regarding liver function (aspartate aminotransferase, alanine aminotransferase, and $\gamma$-glutamyl transpeptidase exceeded standard level) are defined as having hepatic impairment. Information was not available on secondary osteoporosis for 328 patients or on glucocorticoid-induced osteoporosis for 337 patients.

Abbreviation: $\mathrm{n}$, number. 


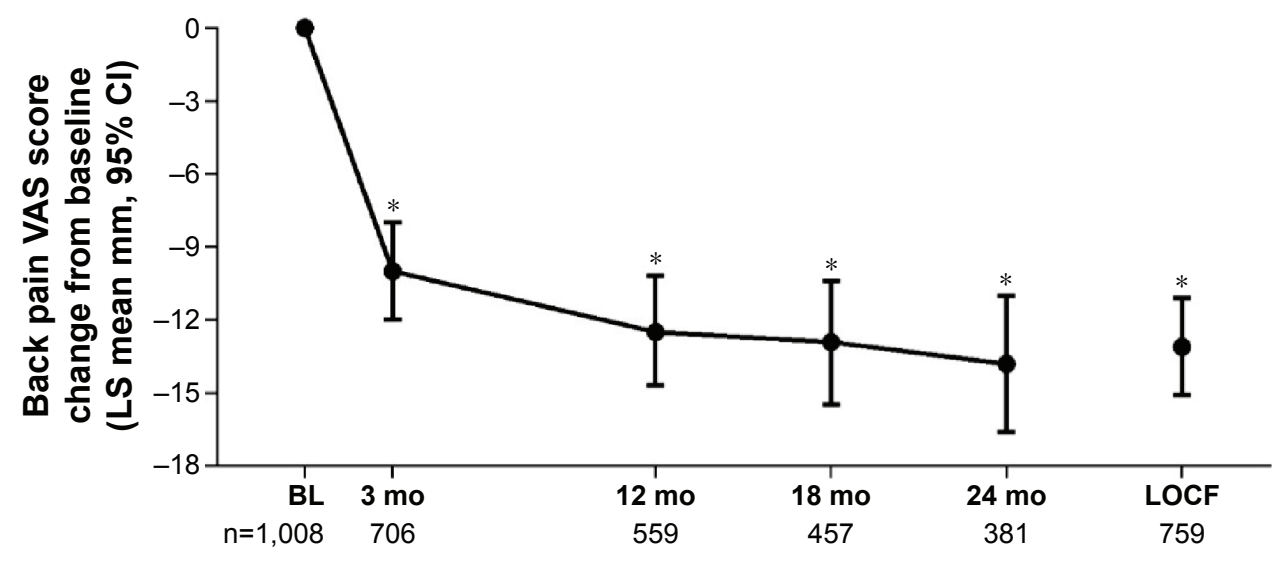

Figure SI LS mean $(95 \% \mathrm{Cl})$ change from baseline in back pain VAS score after 3, 12, 18, and 24 months of teriparatide treatment and at the last observation carried forward in patients with osteoporosis at high risk of fracture in Japan.

Note: $* P<0.001$ for the change from baseline at each time point.

Abbreviations: BL, baseline; $\mathrm{Cl}$, confidence interval; LOCF, last observation carried forward; LS, least squares; VAS, Visual Analog Scale.

A

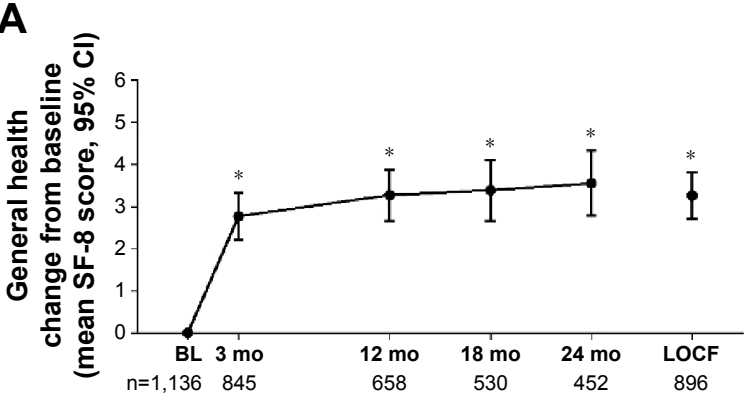

C

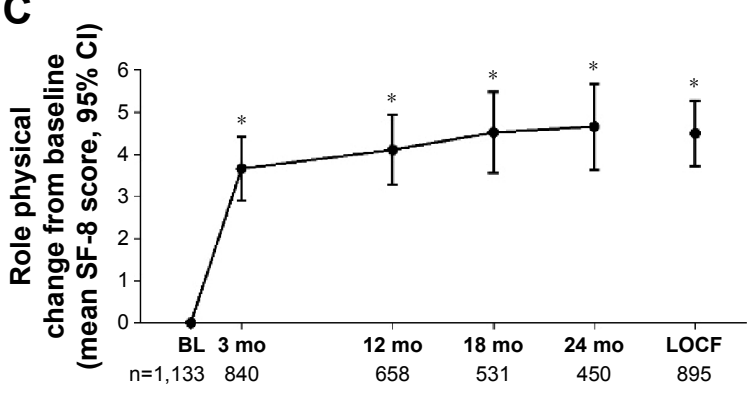

E

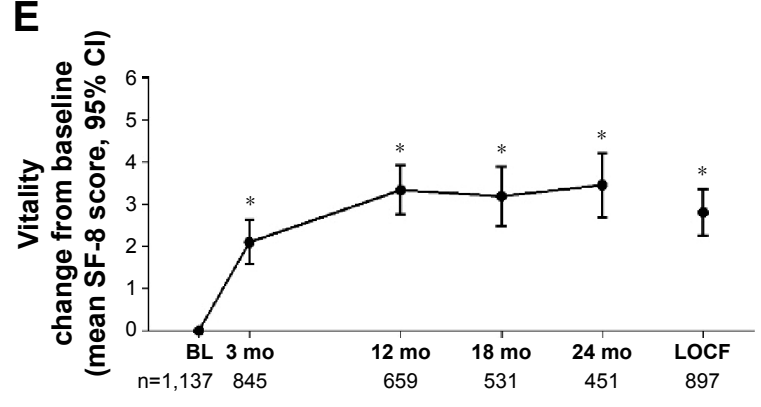

B

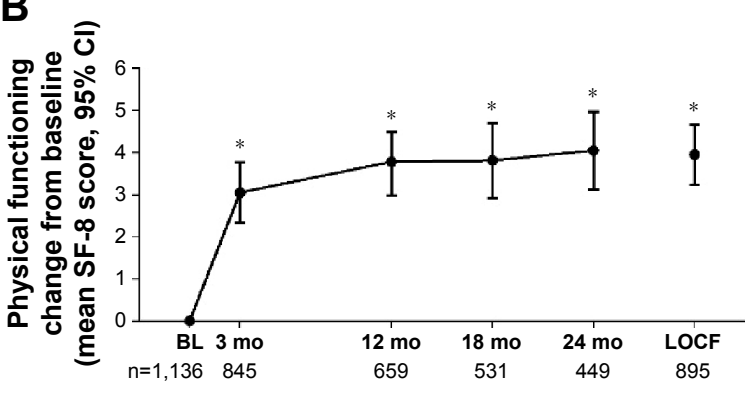

D

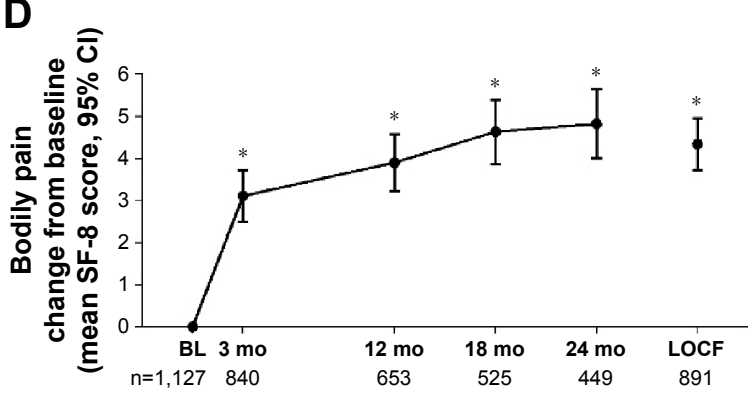

F

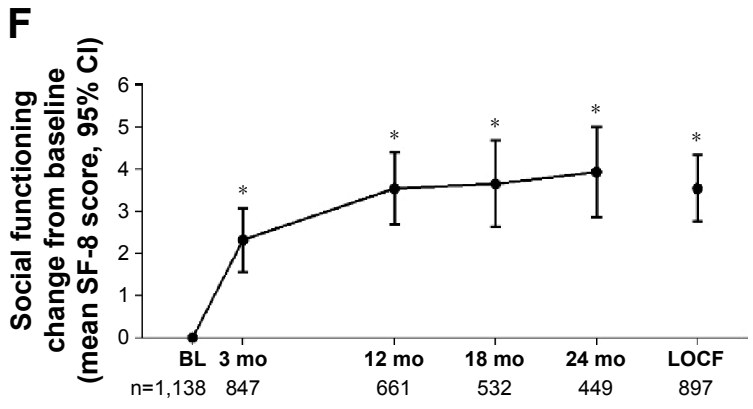

Figure S2 (Continued) 

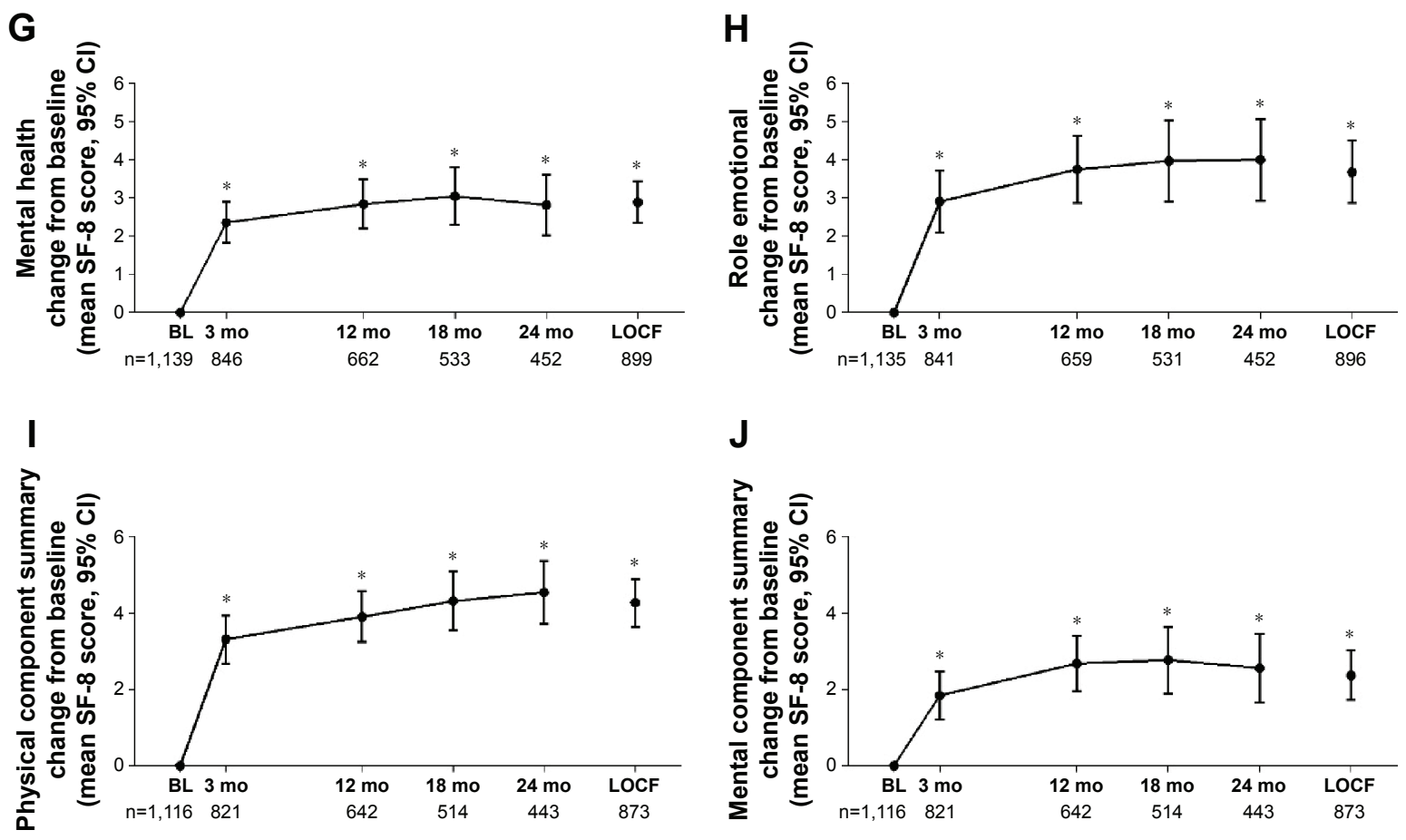

Figure S2 Mean $(95 \% \mathrm{Cl})$ change from BL in SF-8 domains and SF-8 physical and mental component summary scores at 3, I2, 18 , and 24 months of teriparatide treatment, and at the LOCF in patients with osteoporosis at high risk of fracture in Japan.

Notes: $* P<0.00$ I for all SF-8 domains and summary scores at all time points. The mean (SD) BL scores were: (A) general health 44.7 (7.62); (B) physical functioning 39.9 (I0.59); (C) role physical 38.6 (I I.73); (D) bodily pain 4 I.6 (9.09); (E) vitality 45.1 (7.74); (F) social functioning 40.7 (II.09); (G) mental health 46.2 (8.2I); (H) role emotional 42.3 (I I.8I); (I) physical component summary 38.4 (9.32); and (J) mental component summary 45.9 (9.17).

Abbreviations: BL, baseline; $\mathrm{Cl}$, confidence interval; LOCF, last observation carried forward; SD, standard deviation; SF-8, Short Form-8.

\section{Publish your work in this journal}

Clinical Interventions in Aging is an international, peer-reviewed journal focusing on evidence-based reports on the value or lack thereof of treatments intended to prevent or delay the onset of maladaptive correlates of aging in human beings. This journal is indexed on PubMed Central, MedLine,
CAS, Scopus and the Elsevier Bibliographic databases. The manuscript management system is completely online and includes a very quick and fair peer-review system, which is all easy to use. Visit http://www.dovepress. $\mathrm{com} /$ testimonials.php to read real quotes from published authors. 